\title{
On accuracy estimation of ultrasonic thermometry technique with heated wire method
}

\author{
Evgeny Smirnov ${ }^{1}$, Andrey Yukhnev ${ }^{*}$, Yakov Gataulin ${ }^{1}$, Daria Sinitsyna ${ }^{1}$, Alexander \\ Berkovich $^{1}$, and Dmitriy Tarkhov ${ }^{1}$ \\ ${ }^{1}$ Peter the Great St. Petersburg Polytechnic University, 29 Politecnicheskaya St., St. Petersburg, \\ Russia
}

\begin{abstract}
A test object with a heated wire introduced into a tissuemimicking material and a method for determining the coefficient of ultrasonic thermal strain are described. The accuracy of the developed ultrasonic thermometry method is estimated using data of thermistor sensor measurements of time-dependent axisymmetric temperature field around the heated wire.
\end{abstract}

\section{Objectives}

Focused ultrasound is a promising method of human internal tissues heating [1-5]. However, its application is possible only if complete information about the spatial and temporal variations of temperature inside the biological tissue is available. In comparison with the magnetic resonance method currently used in clinics [6], contactless ultrasonic thermometry (UST) has significant advantages in terms of the cost and size of the equipment. The UST method is based on the fact that the speed of sound changes when material temperature varies. This is the reason of a time shift of the ultrasonic signal reflected from the heated material with respect to the reference signal reflected from the unheated one. During the last two decades this method is being developed in a number of scientific centers [7-9]. Animal studies are carried out for approbation of the method [1013]. The sensitivity of the method is evaluated on test objects with known temperature fields [14-15]. High-Intensity Focused Ultrasound (HIFU) [16-17] or a heated wire [15,18] and are used for heating the tissue-equivalent material of test objects. There are some other methods $[23,24,25]$ of thermometry technique which could not be used in our task.

In the present work, we describe the experience in developing a test object with a heated wire and algorithms for processing ultrasonic (US) signals, as well as the initial experience in using the test object for accuracy estimation of the UST temperature measurements inside tissue-mimicking material during its time-dependent heating.

\footnotetext{
${ }^{*}$ Corresponding author: a.yukhnev@mail.ru
} 


\section{Methods}

The test object (Fig. 1) includes a container with a strained nichrome wire $130 \mathrm{~mm}$ long and $0.2 \mathrm{~mm}$ in diameter; thermistor sensors located at different distances from the wire (1 to 6 $\mathrm{mm})$. During the manufacturing process, a container with the wire and the sensors installed in it is filled with tissue-mimicking material (TMM) - agar-agar with graphite filler.

The "reference" axisymmetric temperature field is created by heating and subsequent cooling of the nichrome wire and is measured by thermistor sensors (accuracy is of $0.5^{\circ} \mathrm{C}$ ).

The reflected ultrasonic signal is registered in the scanning plane of a linear 80-element ultrasonic sensor positioned perpendicular to the wire. It is done synchronously with recording the signals from the temperature sensors.

The temperature increment $\Delta T$ at a current point is calculated by estimating the spatial derivative of the ultrasound signal shift, $\Delta d$, by depth $\varepsilon=\frac{d}{d z}(\Delta d)$ and using the following relation: $\Delta T=K \varepsilon$, where $K$ is the material depended ultrasonic thermal strain factor [8].

Note that in a number of works [19,20], the shift is determined by a cross-correlation method. In some works neural networks are used [21,22]. In the present work, an original method was used to estimate the shift of the ultrasound signal. This method operates with the differences in the weighted average values of coordinates for each pair of corresponding half-cycles of the reflected signals from the heated and the unheated material. Tests on model and real signals have shown that this method is quite efficient.

The weighted average of the group center was calculated as $z_{i}=\sum_{k=1}^{N_{i}} A_{k} z_{k} / \sum_{k=1}^{N_{i}} A_{k}$, where $z$ is, as previously, the depth coordinate, $A_{k}$ is the recorded ultrasound signal value, index $\mathrm{k}$ corresponds to a current half-oscillation. The shift itself is calculated as the difference between the weighted averages of the corresponding oscillations $\Delta z_{i}=z_{C_{2 i}}$ $z_{C_{1 i}}$.

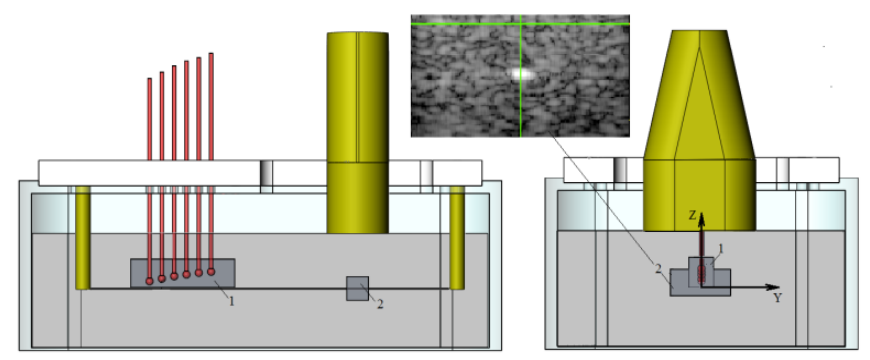

Fig. 1. Test object: 1 - area for temperature measurements, 2 - area for US signal record)

\section{Results}

The thermal shift data for a beam passing near the heated wire $(r=1.8 \mathrm{~mm})$ is shown in Figure 2. 


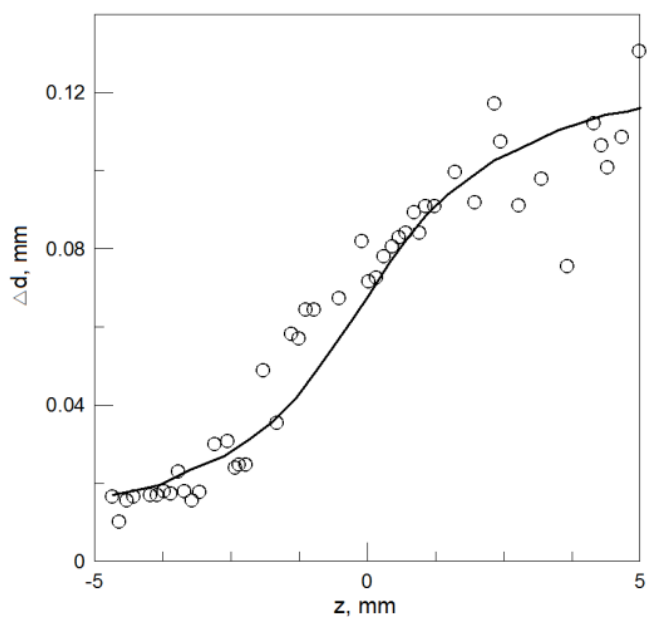

Fig. 2. US signal shift along the beam approximated by arctangent function

Figure 3a shows the thermal strain map obtained using data for all the beams of 161 (at the instant of $25 \mathrm{~s}$ after the wire heating start, $z=0$ corresponds to the center of the wire). Generally, the map corresponds to axisymmetric temperature increase field during the heating of the TMM (Figure 3b).

Figure 4a shows a temperature increase map obtained from the thermal shift strain map (Figure 3a) using $K=530^{\circ} \mathrm{C}$. Experimental data at $|y|<1 \mathrm{~mm}$ is not shown because of strong distortions of the ultrasound field by the wire. As seen, the results obtained with the ultrasound thermometry (Figure 4a) are in a satisfactory agreement with the map reconstructed from the temperature probes measurements (Figure 4b).

Figures 5 and 6 show a comparison of the temperature increase $\Delta T$ determined by ultrasonic thermometry with the one measured by three thermistor sensors (distances from the wire are 1.3, 2.1, and $2.7 \mathrm{~mm}$ ) at instances of 5,15 and $25 \mathrm{~s}$. The maximum difference (about $2.5^{\circ} \mathrm{C}$ ) between data from two methods is observed near the heated wire ( $r=1$ to $1.5 \mathrm{~mm})$. Far away from the wire the difference decreases and does not exceed $1{ }^{\circ} \mathrm{C}$.

a

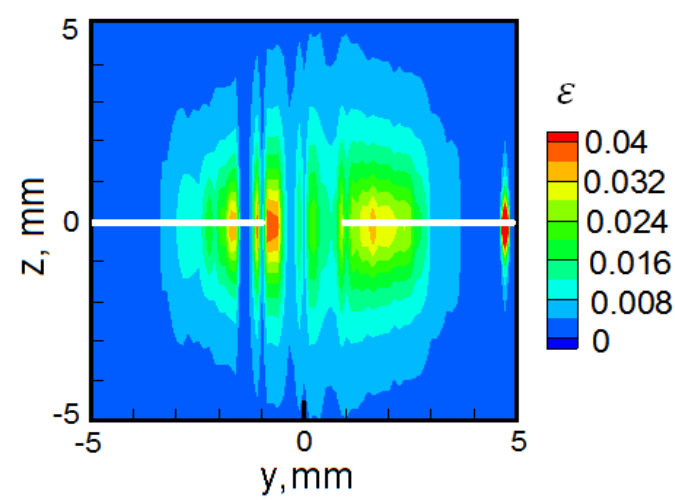

b

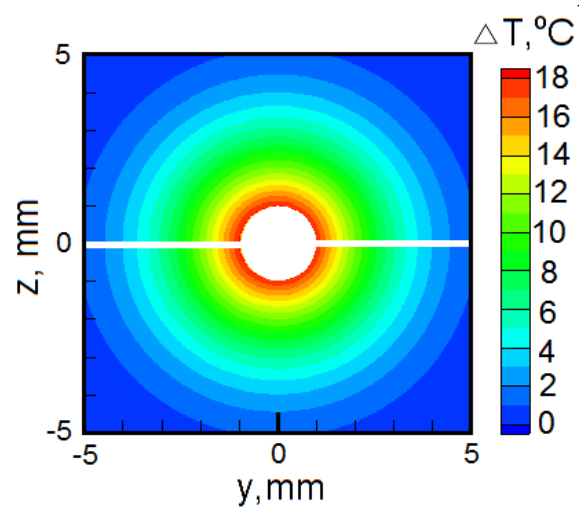

Fig. 3. US thermal shift strain (a) and the axisymmetric field of temperature increase (b) at $t=25 \mathrm{~s}$ (white color indicates the lines of $z=0$ chosen for extraction of data used for the $K$-factor estimation) 

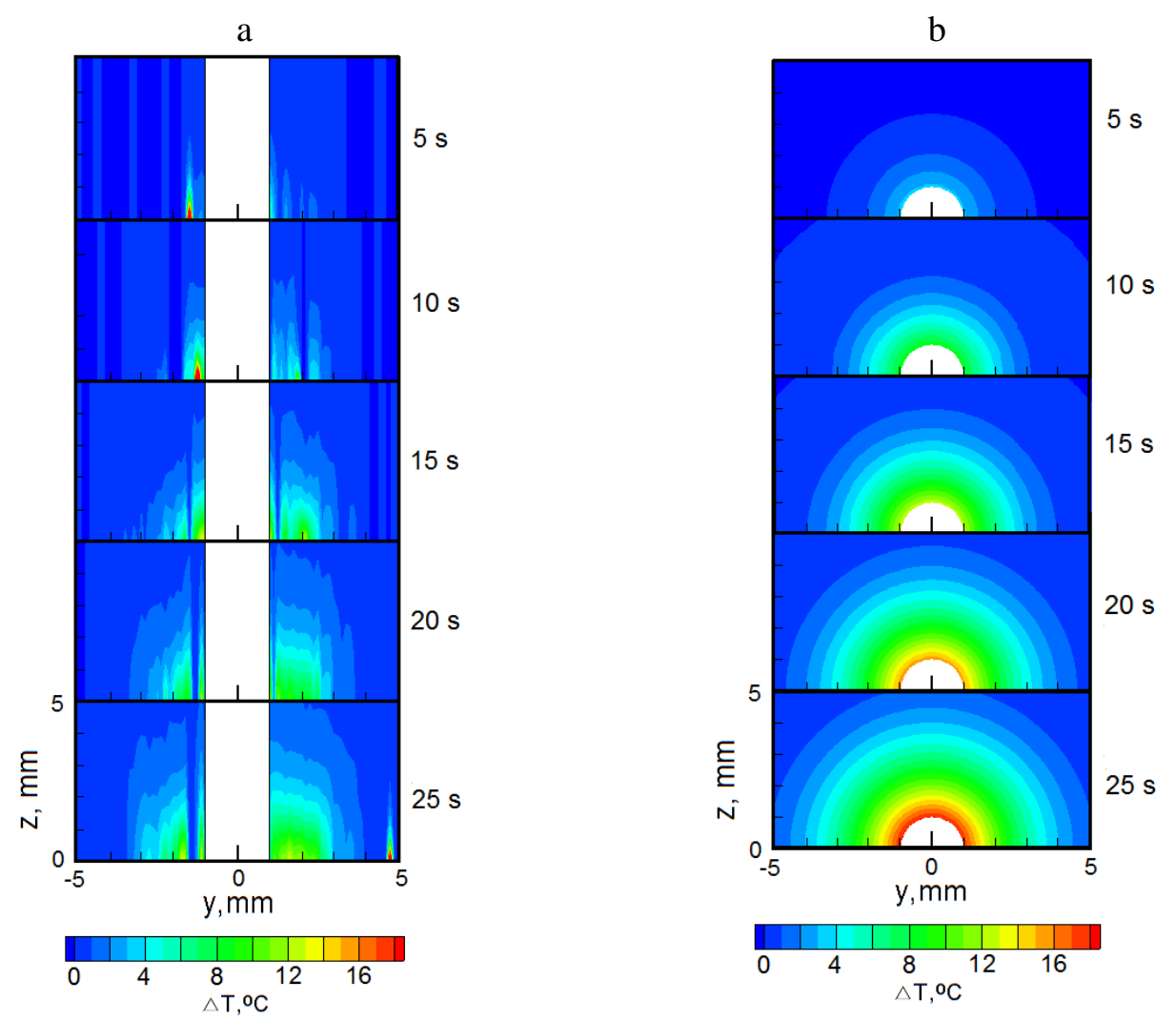

Fig. 4. Spatiotemporal evolution of ultrasound-estimated temperature rise (a) and temperature rise measured by temperature sensors (b)

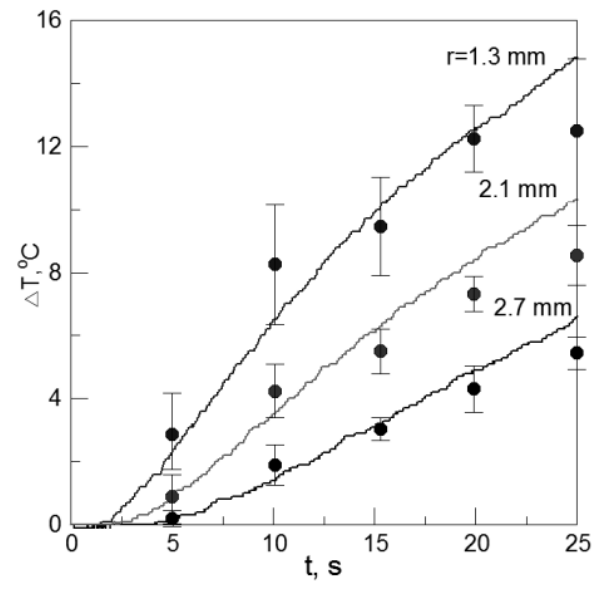

Fig. 5. Temperature increase profiles near the heated wire: (lines) temperature recorded by thermistor sensors, (points) ultrasoundestimated temperature rise averaged over 7 experiments (error bars represent deviation of estimated temperatures)

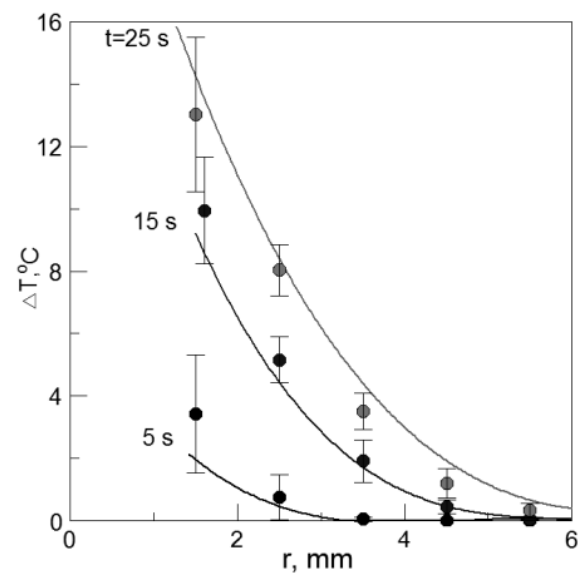

Fig. 6. Temperature increase profiles near the heated wire: (lines) approximating curves of temperature measurements, (points) ultrasoundestimated temperature rise averaged over 7 experiments 


\section{Conclusions}

Time-dependent axisymmetric temperature rise, up to $15{ }^{\circ} \mathrm{C}$ for $25 \mathrm{~s}$, in the tissuemimicking material at a depth of $20 \mathrm{~mm}$ has been measured simultaneously with the ultrasonic thermometry technique and with a set of thermistor sensors. Variations of temperature increase in time and distance from the wire obtained by the two measurement techniques are in a reasonable agreement at distances of more than $1 \mathrm{~mm}$. Overall accuracy of the thermometry technique, as implemented in the developed test-object with a heated wire, is evaluated as 1 to $2{ }^{\circ} \mathrm{C}$ depending on the distances from the wire.

The advantage of the heated wire method for development of the ultrasound thermometry technique is simple axisymmetric the temperature field. The disadvantage is perturbations produced by the wire on the ultrasound signal.

The study was carried out in the framework of the Project No.14.578.21.0263 supported by the Education and Science Ministry of the Russian Federation for 2018-2019.

\section{References}

1. N.N. Petrishchev, D.U. Semenov et al. Patolog. phiz. i exper. terap., 61(2), 46-50 (2017)

2. V.A. Khokhlova, L.A. Crum et al. HIFU Therapy (2017)

3. N.N. Petrishche, D.Y Semyonov, A.Y. Tsibin, A.E. Berkovich et al., Vestnik khirurgii im. I.I.Grekova, 176(5), 101-105 (2017)

4. A.E. Berkovich, A.A. Bursian et al. Biomed. Eng., 51(3), 215-217 (2017)

5. A. Copelan, J. Hartman et al. Semin. Intervent. Radiol., 32, 398-415 (2015)

6. Y. Zhou, Ultrasound Quarterly, 33, 253-260 (2017)

7. E.S. Ebbini, C. Simon, D. Liu, IEEE Signal Process. Mag., 35, 166-74 (2018)

8. P. Karwat, T.Kujawska, et al., Ultrasonics, 65, 211-219 (2016)

9. M.A. Lewis, R. M. Staruch, R.Chopra, Int. J. Hyperthermia, 31(2), 163-181 (2015)

10. A. Haritonova, D. Liu, E.S. Ebbini IEEE Trans. Ultrason., Ferroelec., and Freq. Contr., 62(12), 2031-42 (2015)

11. A.E. Berkovich, A.A. Bursian, K.U. Senchik et al. Biomed. Eng., 50(2), 96-99 (2016)

12. N.N. Petrishchev, A.Y Tsibin, D.Y. Semyonov, A.E. Berkovich et al. Patolog. phiz. i exper. terap., 60(1), 89-93 (2016)

13. M. Bayat, J. R. Ballard, E.S. Ebbini, AIP Conference Proceedings, 1821, 060004 (2017)

14. A. Dabbagh, B.J.J.Abdullah et al., Ultrasonic imaging, 36(4), 291-316 (2014)

15. A.E. Berkovich, E.M. Smirnov, A.D. Yukhnev et al., J. of Physics: Conf. Ser., 1044 (2018)

16. J. Foiret, K.W. Ferrara, PLoS ONE, 10(8), 1-22 (2015)

17. Y.S. Hsiao, C. X. Deng, Med. Biol., 42(2), 503-517 (2016)

18. M. Bayat, J.R. Ballard, E.S. Ebbini, IEEE Trans. on Biomed. Eng., 62(2), 450-57 (2015)

19. R. Maass-Moreno, C.A. Damianou, J. Acoust. Soc. Am., 100(4), 2522-30 (1996)

20. A. Anand, P.J. Kaczkowski, Ultrasound in Med. \& Biol., 34, 1449-64 (2008) 
21. P. Rangraz, H. Behnam, et al., J. of Med. Signals \& Sensors, 2(4), 192-202 (2012)

22. V. Antonov, D. Tarkhov, A. Vasilyev, Math. Models \& Methods in Appl. Sciences, 1-8 (2018)

23. S.E. Aleksandrov, G.A. Gavrilov, A.A. Kapralov, G.Y. Sotnikova, Physics Procedia 73, 177-182 (2015), doi:10.1016/j.phpro.2015.09.151

24. V. Klinkov, V. Aseev, A. Semencha, E. Tsimerman, Sensors and Actuators, A: Physical 277, 157-162 (2018), doi:10.1016/j.sna.2018.04.048

25. A.N. Anisimov, V.A. Soltamov, I.D. Breev, R.A. Babunts, E.N. Mokhov, G.V. Astakhov, V. Dyakonov, D.R. Yakovlev, D. Suter, P.G. Baranov, AIP Advances 8, (2018), doi:10.1063/1.5037158 